\title{
Robotic Picking for Piled Sushi Topping
}

\author{
Kenta Matsuura \\ Graduate School of Engineering Science, Osaka University \\ 1-3 Machikaneyama, Toyonaka 560-8531, Japan \\ Currently with Yaskawa Electric Co. Ltd., Japan \\ Keisuke Koyama, Weiwei Wan, Kensuke Harada \\ Graduate School of Engineering Science, Osaka University \\ 1-3 Machikaneyama, Toyonaka 560-8531, Japan \\ E-mail: \{koyama,wan,harada\}@sys.es.osaka-u.ac.jp
}

\begin{abstract}
This paper proposes a method for picking piled sushi topping. By observing a human motion picking a sushi topping, we propose two picking strategies where one is to insert a finger into the separation among two toppings with shaking the finger, and the other is to insert a finger into the separation between a topping and a table. Along with two segmentation method, i.e., plane segmentation and LCCP segmentation methods, we experimentally verify the effectiveness of the proposed approach.
\end{abstract}

Keywords: Robot, Picking, Food Elastic Object.

\section{Introduction}

In recent years, labor shortage has become a serious problem in the food manufacturing industry, and the demand for automation of food manufacturing has been increasing. For example, taking food out of containers is a monotonous and heavy work for workers, so automation using robots is expected. However, food products are often flexible and irregularly shaped that do not retain a specific shape, such as fish fillets and noodles. This makes difficult to recognize their pose with vision sensors or to grasp them with a robot hand. In this study, we propose a recognition and grasping method focusing on the characteristics of flexible irregular objects, and grasp a sushi topping as an example (Fig.1).

In research on picking industrial parts without deformation, model-based methods[1], visibility-based methods[2], and machine learning-based methods[3]

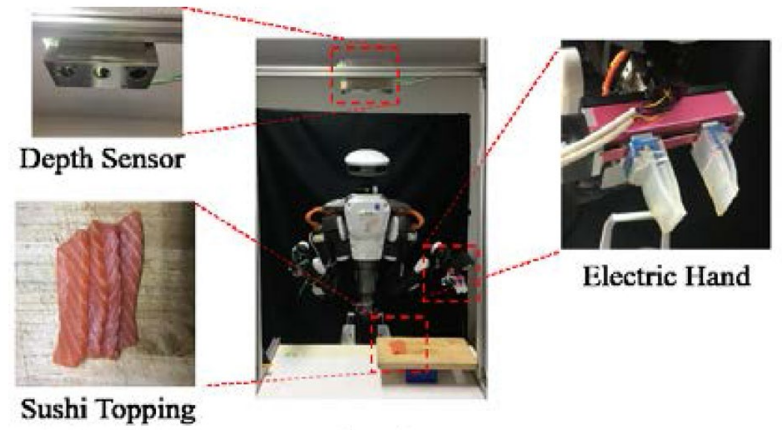

Figure 1 Overview of robotic picking for piled sushi toppings

have been proposed. However, none of them assume flexible food as the object to be grasped. In contrast, in this study, we assume a situation where sushi toppings are stacked, and we consider grasping one of them. When a human grasps a sushi topping, he or she moves his or her fingers from side to side and puts his or her fingers in between the sushi seeds, and grasps one of them by 


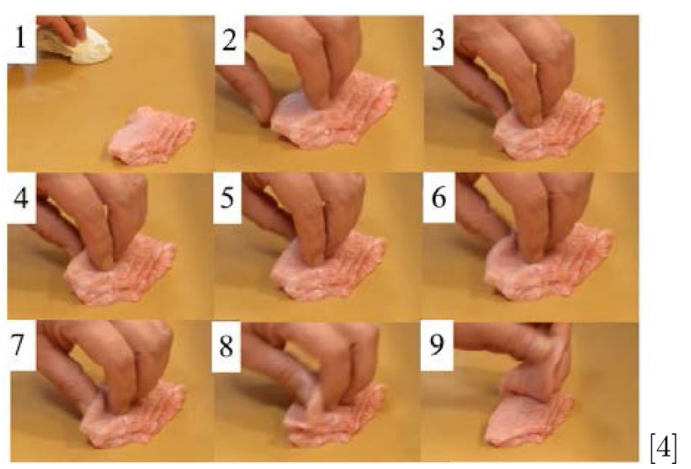

Figure 2 Human strategy for picking piled sushi topping

separating it (Fig. 2). This research examines the possibility of using a robot to perform this kind of grasping task for a flexible object. We consider two segmentation methods of point clouds and two grasping methods based on the human behavior. We evaluate the success rate of each segmentation method and each grasping method by conducting experiments using real sushi toppings.

Section 2 of this paper describes the proposed method, Section 3 describes the experiments and their results, and Section 4 summarizes and discusses future plans.

\section{Proposed Method}

In this study, we use a depth sensor to obtain a point cloud and segment it. To pick a sushi topping, we use a twofingered parallel gripper where each finger is made of soft material. Section 2.1 describes the segmentation method for the point cloud of each sushi species and the calculation of the grasping pose, and Section 2.2 describes the method to grasp one sushi species based on the center of gravity of each segment.

\subsection{Segmentation}

We consider two segmentation methods of captured point cloud. The first method is to detect planes in a 3D point cloud dataset using the RANSAC algorithm and to segment the point cloud into planes [5] (Plane Model Segmentation). The second method is to divide similar voxels into groups of small convex regions, which are separated by concave boundaries[6] (LCCP Segmentation). In this study, after segmentation using Plane Model Segmentation[5] or LCCP Segmentation[6], the center of gravity of a cluster whose size is greater than a threshold is calculated for the purpose of determining the grasping pose. The density of the object was assumed to be uniform, and the center of gravity was calculated by dividing the sum of the $\mathrm{x}, \mathrm{y}$, and $\mathrm{z}$ coordinates of the points belonging to the cluster by the number of points in the cluster for each cluster. The grasped object is selected based on the obtained center of gravity.

\subsection{Grasping method}

In this study, we propose two grasping methods based on the human behavior of grasping sushi toppings. The first method is to insert the finger into the trough between two sushi toppings while shaking the finger to separate them (Fig. 3). The fingertip of the hand is first placed vertically above the separation between the two sushi pieces, and the hand is tilted slightly about the x-axis shown in Fig. 3 , and then moves in the vertically downward direction until the hand hits the sushi piece. Then, the finger is inserted into the trough between the two sushi toppings with shaking the finger. While inserting the finger, the hand is gradually tilted back about the $\mathrm{x}$-axis. The hand grasps the sushi topping after sliding the finger into the sushi sopping.

The second method is to place a finger between the sushi topping and the cutting board (Fig.4). In the coordinate system shown in Fig. 4, the fingertip is placed vertically above the point with the largest $x$-coordinate of the cluster of the largest $\mathrm{z}$-coordinate of the center of gravity. Then, the hand is tilted about the y-axis where its rotation center is located at the fingertip. Then, the hand is moved vertically downward direction until it touches the cutting board. Then, the finger is inserted into the trough between a sushi topping and the cutting board. While inserting the finger, the hand is tilted back about the yaxis. The hand grasps the sushi topping after sliding the finger into the sushi sopping.
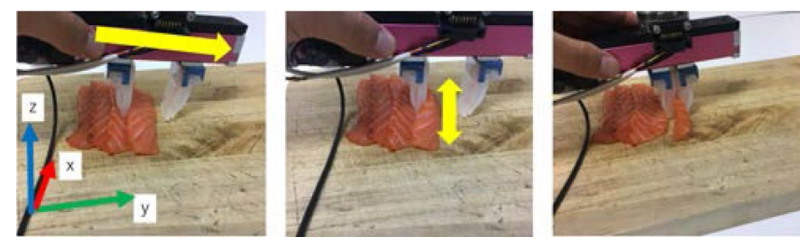

Figure 3 A finger is inserted into the separation between two sushi toppings (Picking strategy 1 ) 

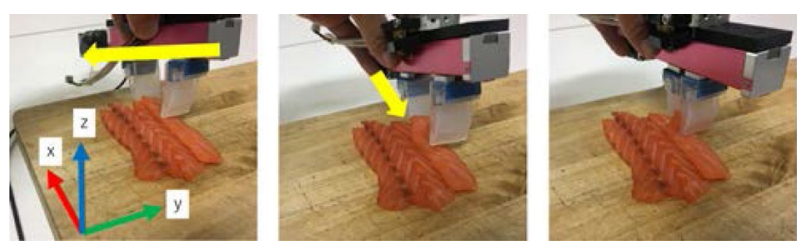

Figure $4 \mathrm{~A}$ finger is inserted between a sushi topping and the chopping board (Picking strategy 2)

\section{Experiment}

In our experiment, we use an industrial manipulator to grasp salmon and scallops, which positions and sizes are unknown as shown in Figure 5(a).

The robot grasps the object based on the information about the position and shape of the object calculated from the obtained point cloud. After acquiring the point cloud, we segment it by using the two methods described in Section 2.1. After segmentation, we calculate the center of gravity of the cluster with more than 100 points. The robot picks the object for 10 times by using two segmentation methods and two grasping methods.

The hand used in the experiment is an electric hand manufactured by THK Corporation where its fingers are made of silicon that allows the fingertips to maintain a moderate stiffness for grasping food (Fig. 5). The dimensions of a finger are $45 \mathrm{~mm}$ in length, $20 \mathrm{~mm}$ in width, $30 \mathrm{~mm}$ in opening and closing width, and $6 \mathrm{~mm}$ in thickness of the fingertip. We used salmon and scallops, and the scallops were opened and laid out on the table in front of the robot.

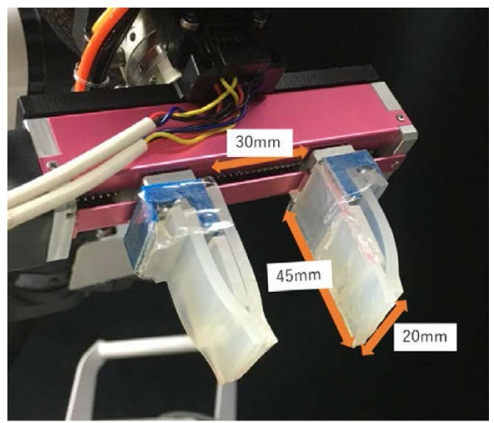

Figure 5 Electric hand used in our experiment

\subsection{Experimental Results and Discussions}

In Figs. 6 and 7, (a) shows the sushi toppings arranged on the cutting board, (b) shows the result of segmenting (a) into clusters by using the Plane Model Segmentation where points with the same color belong to the same cluster, and (c) shows the result of segmenting (a) into clusters by using the LCCP Segmentation. Fig. 8 shows the experimental result using the grasping method 1 , and Fig. 9 shows the result of using the grasping method 2 . The number of successful grasps out of 10 trials with two different segmentation methods is shown in Table 1.

In Plane Model Segmentation, as the distance between two bumps became small, it often becomes impossible to segment each sushi piece accurately. This is due to the unevenness and curvature of the sushi species since this method divides the point cloud into planes. Therefore, we could not successfully grasp the object when the separation between two pieces was $10 \mathrm{~mm}$ or $15 \mathrm{~mm}$. On the other hand, LCCP Segmentation succeeded in segmenting the region with high accuracy. In the Plane Model Segmentation, we adjusted the thickness of each cluster. On the other hand, in LCCP Segmentation, we adjusted the angle at which the object was considered to be concave. The grasping method 1 was able to grasp all the sushi species at all distances between two pieces if they were divided into appropriate clusters. Even if there was almost no gap in the trough between two sushi species, it was sometimes possible to grasp them by inserting the fingers while moving it back and forth. Grasping method 2 was successful when the distance between two pieces was $15 \mathrm{~mm}$ and $20 \mathrm{~mm}$. When the distance was $10 \mathrm{~mm}$, the finger interfered with the neighboring sushi topping and could not insert the finger between the sushi topping and the cutting board. In both grasping methods 1 and 2, the weight of the scallop was larger than that of the salmon, so the scallop was sometimes dropped even after grasping it once if it was grasped too shallowly. 


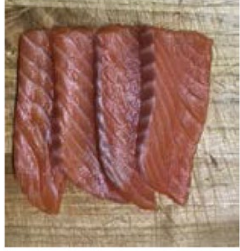

(a) Sushi toppings

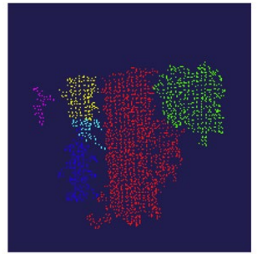

(b) Plane Model Segmentation

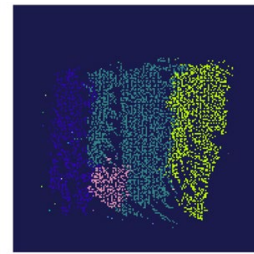

(c) LCCP

Segmentation

Figure 6 Result of segmentation (Salmon)

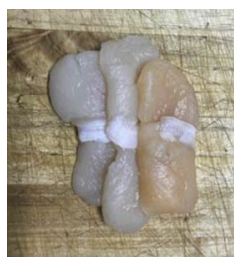

(a) Scallops

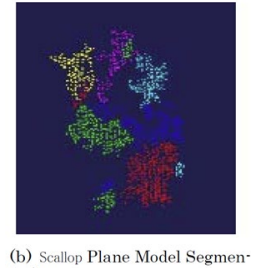

tation

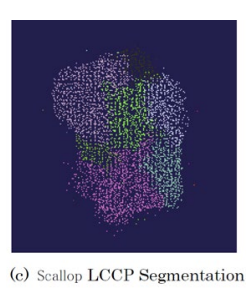

Figure 7 Result of segmentation (Scallop)

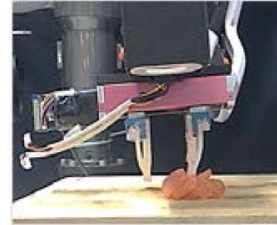

(a) Initial position

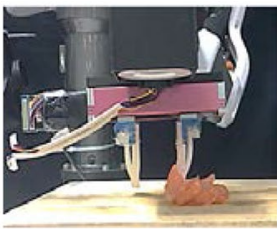

(b) Insert

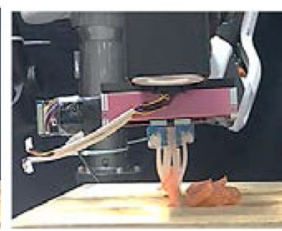

(c) Grasp
Figure 8 Result of picking strategy 1

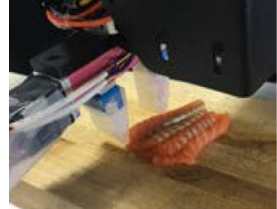

(a) Initial position

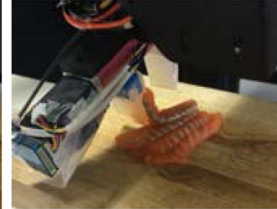

(b) Insert

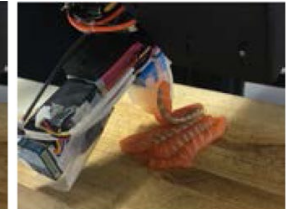

(c) Grasp
Figure 9 Result of picking strategy 2

Table 1 Number of successful picking

\begin{tabular}{|c|l||r|r|r||r|}
\hline strategy & segmentation & $\begin{array}{c}\text { salmon } \\
10[\mathrm{~mm}]\end{array}$ & $\begin{array}{c}\text { salmon } \\
15[\mathrm{~mm}]\end{array}$ & $\begin{array}{c}\text { salmon } \\
20[\mathrm{~mm}]\end{array}$ & $\begin{array}{c}\text { scallop } \\
15[\mathrm{~mm}]\end{array}$ \\
\hline \hline 1 & PMS & 0 & 0 & 5 & 0 \\
\hline 1 & LS & 6 & 6 & 7 & 4 \\
\hline 2 & PMS & 0 & 0 & 4 & 0 \\
\hline 2 & LS & 0 & 6 & 5 & 4 \\
\hline
\end{tabular}

\section{Conclusions}

In this study, we proposed a unique grasping method for grasping flexible irregular objects by using two different methods to recognize objects from point cloud information and to find the center of gravity of clusters. From the experiments, we confirmed that when the objects are lined up with flexible irregular shapes, such as the sushi species used in this study, LCCP Segmentation can provide robust recognition, and the grasping method 1 is relatively flexible regardless of the spacing of the objects.

In the future, we will develop the segmentation method used in this study to further improve the accuracy of segmentation and to estimate not only the center of gravity but also the approximate size and angle of the objects from the point cloud. In addition, we are investigating the construction of a system that can flexibly grasp various types and arrangements of objects instead of a fixed placement method.

\section{References}

1. K. Harada, W. Wan, T. Tsuji, K. Kikuchi,K. Nagata, and H. Onda. Iterative visual recognition for learning based randomized bin-picking. Experimental Robotics, pp. 646655. Springer, 2016.

2. Y. Domae, H. Okuda, Y. Taguchi, K. Sumi, and T. Hirai. Fast graspability evaluation on single depth maps for bin picking with general grippers. IEEE Int. Conf. on Robotics and Automation (ICRA), pp. 1997-2004. 2014.

3. R. Matsumura, Y. Domae, W. Wan, and K. Harada, Learning Based Robotic Bin-picking for Potentially Tangled Objects, IEEE/RSJ Int. Conf. on Intelligent Robots and Systems, pp. 7984-7991, 2019.

4. Sushi-master Toshi, https://youtu.be/QgcxLLZ7KJs, Jan., 2019.

5. A. Hast, J. Nysjo, and A. Marchetti. Optimal ransactowards a repeatable algorithm for finding the optimal set. 2013.

6. S. Christoph Stein, M. Schoeler, J. Papon, and F. Worgotter. Object partitioning using local convexity, IEEE Conference on Computer Vision and Pattern Recognition, pp. 304-311, 2014.

7.

(C) The 2021 International Conference on Artificial Life and Robotics (ICAROB2021), January 21 to 24, 2021 